\title{
Benchmarks
}

nomics 13:718-725.

11.Van der Ploeg, L., A. Liu and P. Borst. 1984. Structures of the growing telomeres of trypanosomes. Cell 36:459-468.

12.Weber, B., C. Collin, C. Robbins, R.E. Magenis, A.D. Delaney, J.W. Gray and M.R. Hayden. 1990. Characterisation and organisation of DNA sequences adjacent to the human telomere associated repeat (TTAGGG) $)_{n}$. Nucleic Acids Res. 18:3353-3361.

13.Whitcomb, J.M., A. Rashtchian and S.H. Hughes. 1993. A new PCR based method for the generation of nested deletions. Nucleic Acids Res. 17:4143-4146.

14.Wicky, C., A.M. Villeneuve, N. Lauper, L. Codourey, H. Tobler and F. Muller. 1996 Telomeric repeats (TTAGGC) $)_{n}$ are sufficien for chromosome capping function in Caenorhabditis elegans. Proc. Natl. Acad. Sci. USA 93:8983-8999.

We are grateful to Susan Brewster, Bronwen Lambson and Sharon McCann for critically reading the manuscript and helpful discussions. This work was supported by a grant from the Medical Research Council (UK). Address correspondence to Dr. Douglas C. Barker, MRC Outstation of NIMR, Molteno Laboratories, Department of Pathology, University of Cambridge, CB2 1QP, Cambridge, England, UK. Internet: dcb12@mole.bio.cam.ac.uk

Received 22 September 1997; accepted 4 December 1997. Guoliang Fu and Douglas C.
Barker

University of Cambridge

Cambridge, England, UK

\section{PCR-Assisted cDNA Cloning: A Guided Tour of the Minefield}

BioTechniques 24:390-392 (March 1998)

cDNA cloning based on partial or degenerate sequence information is an essential part of molecular biology. In the past few years, polymerase chain reaction (PCR)-assisted cloning techniques (random amplification of cDNA ends [RACE], reverse transcription [RT]-PCR) have been used extensively to determine the $5^{\prime}$ and $3^{\prime}$ ends of cDNA sequences and also (using degenerate oligonucleotides) to identify new members of emerging gene families. Nevertheless, PCR's unique character also engenders some specific problems (5). The case study described here is a cautionary tale that illustrates the most prominent of these. A detailed description of the techniques used is presented elsewhere (4).

\section{Pitfall 1: Skewed Tissue Expression Information}

Degenerate oligonucleotides were designed to amplify from B lymphocytes novel metalloprotease members with a disintegrin and metalloprotease domain (ADAMs) (6). Among the 35 fragments of approximately $200 \mathrm{bp}$ with an open reading frame (ORF) obtained, one was a novel ADAM member (ADAM 20) and was further studied. Only much later (see Pitfall 4) did it become apparent that this novel cDNA is in fact expressed mainly in testis (Figure 2D). This is the only pitfall with a narrow silver lining, in that RT-PCR allows finding novel cDNAs even when starting from the wrong tissue. Unbiased gene expression data can be obtained using quantitative RT-PCR or RNase protection protocols.

\section{Pitfall 2: RACE Tends to Yield Short Products}

B-cell or placenta cDNA was linkerligated, and $5^{\prime}$ and $3^{\prime}$ ends were amplified using a cDNA-specific primer from the novel 200-bp sequence and a linker oligonucleotide using the Marathon $^{\mathrm{TM}}$ cDNA Synthesis Kit (Promega, Madison, WI, USA). Newly obtained sequence was then used to repeat the process with more distally 5' - or 3'-located oligonucleotides. Although each time the longest PCR products were selected for subcloning and sequencing, the PCR apparently strongly favors the shortest products the oligonucleotides allow to amplify. As shown in Figure 1, this method yielded fragments of $1 \mathrm{kbp}$ at most per amplification.

One solution for this problem is to ensure that the starting RNA for the cDNA synthesis is full length (size fractionation may help). Another possi- bility is to serially dilute the linkerligated cDNA samples for the PCR, so that truncated and full-length cDNA samples do not end up in the same reaction vessel.

\section{Pitfall 3: Mutations}

Under the best circumstances, the mutation rate of $T a q$ DNA polymerase was reported to be around $10^{-5}(1-3)$. In this project, however, the mutation rate was at least an order of magnitude higher, at about 2-3 mutations per 400-bp readout $\left(6 \times 10^{-3}\right)$. In most cases, comparison of several cloned RACE products identified a clear majority of (probably) original vs. mutated sequences, but occasionally there was an approximately $50 \%$ split between two groups of variant sequences, presumably because a mutation had occurred early on in the amplification. These mutations were artifactual because they were not seen in multiple clones obtained from a $\lambda$ library of testis cDNA (4). After the RACEs, the Titan ${ }^{\mathrm{TM}}$ High Fidelity PCR System (Boehringer Mannheim, Indianapolis, IN, USA) was used to amplify full-length cDNA; however, this procedure also failed to yield completely mutation-free products.

\section{Pitfall 4: Nonspecific Co-amplifica- tion}

A full-length RT-PCR product was gel-purified (Figure 2A) and used as probe in multiple-tissue Northern blot hybridizations (Figure 2C) and for the screening of a placenta cDNA $\lambda$ library (placenta tissue had shown a good Northern signal; Figure 2C). Figure 2B shows a filter from the secondary screening of a positive $\lambda$ clone with strongly hybridizing plaques. Surprisingly, these positive phages subsequently failed to hybridize with a probe that was prepared from the same fragment after it had been subcloned into a plasmid. When the Northern blots were re-probed with this subcloned fragment, a unique, testis-specific signal was observed (Figure 2D). What apparently had happened was that the RTPCR had co-amplified a ubiquitously expressed cDNA and that this product had co-migrated with the major ADAM band (Figure 2A). This contaminating 
fragment must have produced the ubiquitous signal in Figure $2 \mathrm{C}$ as well as the positive plaques shown in Figure 2B. The testis-specific band could also be seen in Figure 2C (arrow). Such a probe contamination cannot be eliminated by gel purification and another PCR. The solution here is to use a nested set of oligonucleotides.

\section{Pitfall 5: Hybrid Products}

One of the $5^{\prime}$ RACE products was identical to the $5^{\prime}$ sequence of expressed sequence tag (EST) cDNA Clone No. 231995 (Research Genetics, Huntsville, AL, USA). Its 5' end was re-sequenced. This showed that the similarity to ADAM cDNA abruptly stops at some point and that the RACE product was a hybrid sequence of the EST and ADAM cDNAs. This amplification artifact probably arose because of chance sequence similarity between the two cDNAs.

Many of these pitfalls might have manifested themselves here prominently because the cDNA fragments were amplified from sources (lymphocytes,

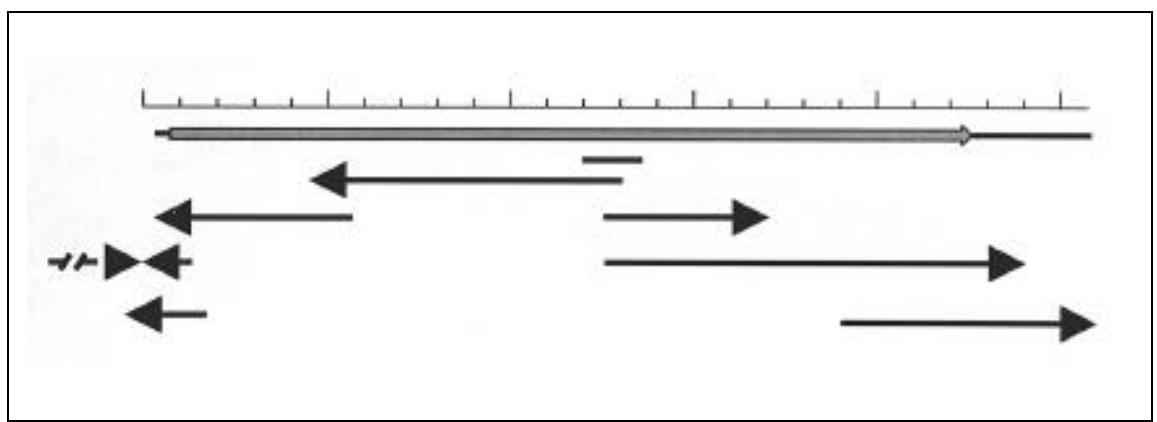

Figure 1. Organization of RACE products obtained (black arrows), starting from a small fragment (black line) of known sequence. The ORF of the corresponding cDNA (gray arrow; total length $2.4 \mathrm{kbp}$ ) and untranslated regions are indicated. Double arrow: hybrid extension product (Pitfall 5). The size bar (top) is divided into 100-bp units.

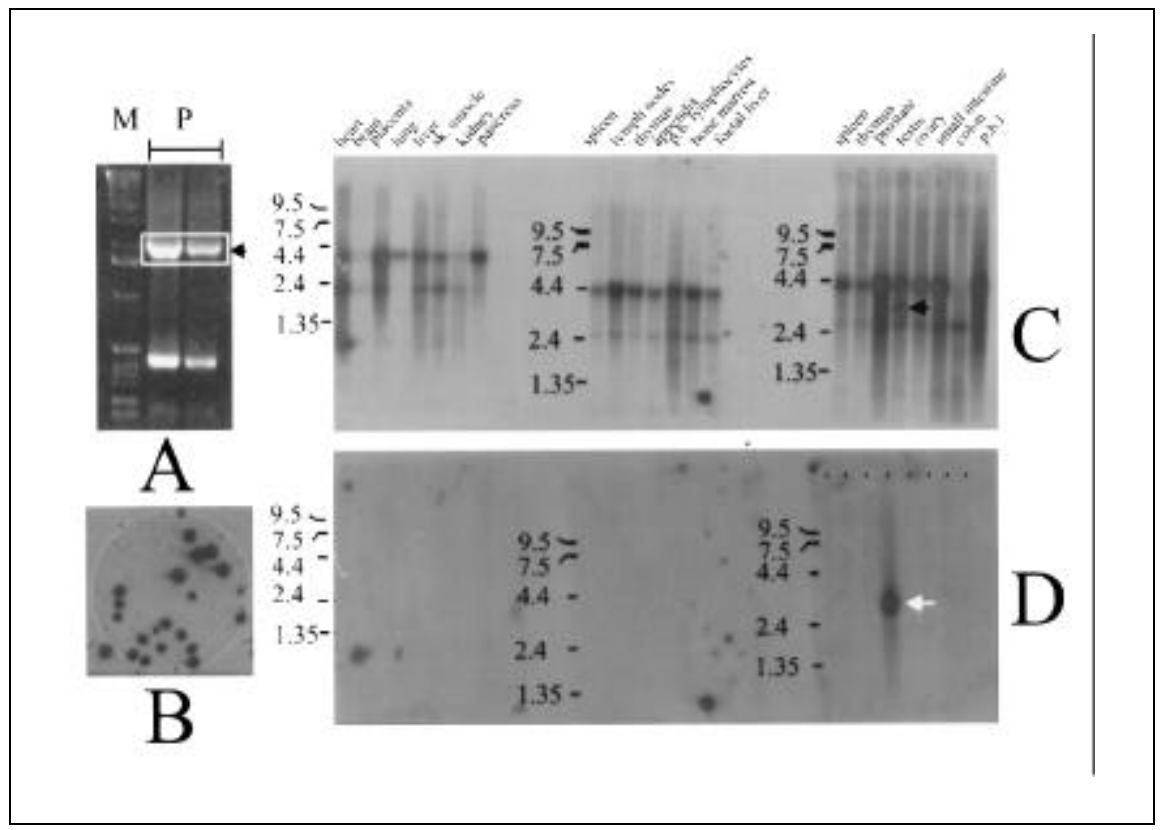

Figure 2. (A) Agarose RT-PCR product (P) of full-length ADAM 20 cDNA. The boxed area was excised for fragment isolation and probe preparation. Lane M: size markers (1-kb ladder; Life Technologies, Gaithersburg, MD, USA). (B) Second-round screening by hybridization of a positive $\lambda$ colony using this full-length fragment from Panel A as a probe. (C) Multiple-tissue Northern blots probed with the same probe as for Panel B. (D) Same blots as in Panel C, probed with the RT-PCR product after subcloning and repurification. 
placenta) in which the ADAM mRNA was extremely rare (Figure 2D). This is probably the origin of the observed high mutation rate and the artifactual amplification of hybrid products (Pitfalls 3 and 5). The correct tissue distribution of the cDNA under study was obscured because of Pitfall 4. A clear lesson from this work is that full-length cDNA cloning must involve library screenings for as much as possible of the coding sequence and that RACE or RT-PCR protocols, if used at all, should be limited to obtain sufficient sequence for hybridizations or for the elucidation of the less critical untranslated regions.

It is well-known that library cDNA clones also often contain extraneous sequences, mostly in the form of unspliced exons, but these are nearly always derived from the same gene. Usually, only a single clone is found per aberrant construct.

Subcloned, gel-purified DNA fragments are usually contaminated with the plasmid DNA from which they had been excised. When used as a probe, these contaminations often manifest themselves by their strong hybridization to the size markers. These contaminations are usually harmless in Northern blot hybridizations or library screenings (except when plasmid libraries are screened). However, RTPCR-generated contaminations such as those described in Pitfall 4 are far more insidious and should be avoided at all times.

\section{REFERENCES}

1.Cline, J., J.C. Braman and H.H. Hogrefe. 1996. PCR fidelity of $P f u$ DNA polymerase and other thermostable DNA polymerases. Nucleic Acids Res. 24:3546-3551.

2.Eckert, K.A. and T.A. Kunkel. 1990. High fidelity DNA synthesis by the Thermus aquaticus DNA polymerase. Nucleic Acids Res. 18:3739-3744.

3.Erlich, H.A., D. Gelfand and J.J. Sninsky. 1991. Recent advances in the polymerase chain reaction. Science 252:1643-1651.

4.Hooft van Huijsduijnen, R. 1998. ADAM 20 and 21; two novel human testis-specific membrane metalloproteases with similarity to fertilin- $\alpha$. Gene 206:273-282.

5.Kenzelmann, M. and K. Mühlemann. 1997. Pitfalls of PCR: cross-reactivity with joyride E. coli nucleic acid. BioTechniques 23:204206.

6.Wolfsberg, T.G., P.D. Straight, R.L. Gerena, A.P. Huovila, P. Primakoff, D.G. Myles and
J.M. White. 1995. ADAM, a widely distributed and developmentally regulated gene family encoding membrane proteins with a disintegrin and metalloprotease domain. Dev. Biol. 169:378-383.

Address correspondence to Rob Hooft van Huijsduijnen, Serono Pharmaceutical Research Institute (formerly Geneva Biomedical Research Institute), 14, chemin des Aulx, Case Postale 674, 1228 Plan-lesOuates/Geneva, Switzerland. Internet: rob. hooft@serono.com

Received 28 July 1997; accepted 4 November 1997.

\section{Rob Hooft van Huijsduijnen Serono Pharmaceutical Research Institute Geneva, Switzerland}

\section{Use of the Restriction Enzyme AvaI and Exo-Bst Polymerase in Strand Dis- placement Amplification}

BioTechniques 24:392-396 (March 1998)

Strand displacement amplification (SDA) is an isothermal in vitro method of amplifying DNA (10-12). This technique is based on the ability of a restriction enzyme to nick one strand of a hemiphosphorothioated form of its double-stranded recognition site and the ability of a polymerase to initiate synthesis at the nick and displace the downstream DNA strand during replication. The method consists of two parts: (i) a target generation process that makes copies of the target sequence flanked by nickable restriction sites and (ii) the exponential amplification of these modified target sequences by repeated nicking, strand displacement and priming of displaced strands, as depicted in Figure 1. The first SDA system we developed used the restriction enzyme HincII and the $3^{\prime} \rightarrow 5^{\prime}$ exonuclease-deficient Klenow fragment of E. coli polymerase I (exo- Klenow). This system achieves $10^{8}$-fold amplification in $2 \mathrm{~h}$ at $37^{\circ}-40^{\circ} \mathrm{C}(10-13)$. More recently, we have developed a thermophilic SDA system that operates at $60^{\circ} \mathrm{C}$ and uses a restriction enzyme from Bacillus stearothermophilus $(B s o \mathrm{BI})$ and a $3^{\prime} \rightarrow 5^{\prime}$ exonuclease-deficient Klenow fragment of a DNA polymerase from $B$. caldotenax (exo- $B c a$ ) (7). This system is faster and more powerful, achieving a $10^{10}$-fold amplification in $15 \mathrm{~min}$. It is also more specific, with a significant reduction in background amplification.

The restriction enzyme AvaI from the blue-green algae Anabaena variabilis (3) is an isoschizomer of BsoBI. Previously, we had preliminarily demonstrated SDA at $37^{\circ} \mathrm{C}$ (12) using $A v a I$ and the $3^{\prime} \rightarrow 5^{\prime}$ exonuclease-deficient Klenow-type fragment of a DNA polymerase from $B$. stearothermophilus ( $\mathrm{exo}^{-}$ $B s t$ ) (1). In the present study, we recombined $A v a \mathrm{I}$ and exo- $B s t$ at $50^{\circ}-55^{\circ} \mathrm{C}$, testing their ability to amplify a target sequence in the IS6110 insertion-like 